\section{Metastatic tumour of right atrium mimicking constrictive pericarditis and tricuspid stenosis}

Though secondary cardiac tumours are 20 times more common than primary lesions, ${ }^{1}$ and cardiac metastases are found in up to $19 \%$ of necropsies undertaken on patients who have died of cancer, ${ }^{2}$ correct diagnosis of secondary spread to the heart is rare. We describe a patient in whom an unrecognised right atrial metastasis was clinically mistaken for constrictive pericarditis while results of cardiac catheterisation suggested tricuspid stenosis.

\section{Case report}

A 54-year-old Kenyan man presented with tiredness and dyspnoea of two months' duration. He was wasted, afebrile, and had pronounced ascites and leg oedema. His pulse was 76 beats/min, regular, of low volume and not paradoxical, and blood pressure was $130 / 80 \mathrm{~mm} \mathrm{Hg}$. The jugular venous pressure was raised to the angle of the jaw and no abnormal pulsation was noted. The heart sounds were quiet, a third sound was present, and on two isolated occasions a soft, low-pitched, mid-diastolic murmur was heard at the left lower sternal border. The liver was enlarged to four finger breadths below the right costal margin. A chest $x$-ray film showed apical opacification and cavitation in both lungs, with a normal heart size. The electrocardiogram showed generalised low voltage. Although acid-fast bacilli were not detected, pulmonary tuberculosis and constrictive pericarditis were diagnosed, and antituberculous and diuretic treatment was started. Cardiac catheterisation (table) showed a pressure gradient across the tricuspid valve suggesting tricuspid stenosis. While awaiting cardiac surgery the patient's condition deteriorated and he died.

Measurements of intracardiac pressure

\begin{tabular}{lccc}
\hline & Phasic & Mean & Comment \\
\hline Right atrium & $\mathrm{a}=21 \mathrm{~mm} \mathrm{Hg} ; \mathrm{v}=21 \mathrm{~mm} \mathrm{Hg}$ & $16 \mathrm{~mm} \mathrm{Hg}$ & Raised \\
Right ventricle & $16 / 5 \mathrm{~mm} \mathrm{Hg}$ & Normal \\
Pulmonary artery & $22 / 10 \mathrm{~mm} \mathrm{Hg}$ & $15 \mathrm{~mm} \mathrm{Hg}$ & Normal \\
Pulmonary wedge & $95 / 5 \mathrm{~mm} \mathrm{Hg}$ & $10 \mathrm{~mm} \mathrm{Hg}$ & Normal \\
Left ventricle & $95 / 65 \mathrm{~mm} \mathrm{Hg}$ & $75 \mathrm{~mm} \mathrm{Hg}$ & Low \\
Aorta & & & \\
\hline
\end{tabular}

Necropsy showed bilateral pulmonary tuberculosis and congestive cardiac failure. A firm, greyish, round tumour, $10 \times 7 \times 6 \mathrm{~cm}$, was present in the righ atrium lateral to the coronary sinus. The bulk of the tumour was above the tricuspid valve but it infiltrated through all the layers of the heart, including the valve's lateral cusp. A small right para-adrenal tumour nodule was found, but no other lesions were detected. Histological examination of the two tumours showed the features of infiltrating, undifferentiated carcinoma. Both were clearly metastatic lesions but their primary source could not be determined.

\section{Comment}

In general, the clinical effects of any cardiac tumour depend more on its site than on its pathological nature. The most common manifestations of cardiac metastases are pericarditis and pericardial effusions, arrhythmias, and heart failure. ${ }^{1}$ Carcinoma of the breast, bronchus, and kidney and melanoma, leukaemia, and lymphoma are the most frequently reported primary lesions, ${ }^{2}$ though in sub-Saharan Africa metastases from hepatocellular carcinoma, Kaposi's sarcoma, and Burkitt's lymphoma assume a greater importance. ${ }^{3}$

A secondary deposit confined to one of the heart chambers behaves essentially like a myxoma. When right-sided, such lesions may cause right heart failure, tricuspid murmurs, low-voltage electrocardiograms, and pressure gradients across the tricuspid valve suggestive of tricuspid stenosis. The clinical and haemodynamic findings of right atrial myxoma and constrictive pericarditis may also resemble each other closely. ${ }^{5}$ Other possible misdiagnoses of a right atrial tumour include cor pulmonale, thromboembolic pulmonary hypertension, and, in patients receiving treatment for known malignant disease, cardiac side effects from cytotoxic drugs.

The case reported here is of interest because of the perplexing physical and haemodynamic findings, but also as a reminder that meta- static heart disease is more common than is generally realised. Patients with certain malignant diseases now live longer and often receive drugs that may themselves cause cardiac side effects. It is important to remember the possibility of the spread of tumour to the heart.

1 McAllister HA, Fenoglio JF. Tumors of the cardiovascular system. Washington DC: Armed Forces Institute of Pathology, 1978.

2 Young JM, Goldman IR. Tumor metastasis to the heart. Circulation $1954 ; 9: 220-9$.

3 Lothe F, Somers K. Secondary tumors of the heart and pericardium in Ugandan Africans. Archives of Pathology 1960;69:158-67.

4 Soulié P, Acar J, Renault P, Lainée R, Azoulay. Les myxomes de l'oreillette droite. Arch Mal Coeur 1961;54:241-73.

5 Emanuel RW, Lloyd WE. Right atrial myxoma mistaken for constrictive pericarditis. Br Heart $\mathcal{F} 1962 ; 24: 796-800$.

(Accepted 18 August 1982)

Kenyatta National Hospital, University of Nairobi, PO Box 30588, Nairobi, Kenya

K M De COCK, MRCP, DTM\&H, lecturer in medicine (now: research fellow, unit of geographical pathology, St Thomas's Hospital Medical School, London)

D K GIKONYO, MB, MMED, lecturer in medicine

S B LUCAS, MRCP, MRCPATH, senior lecturer in human pathology (now : honorary senior lecturer, department of histopathology, St Thomas's Hospital, London)

Clinical Research Centre, PO Box 20778, Nairobi, Kenya

J B O WERE, MB, MMED, research fellow

\section{Photophobia in lithium intoxication}

The neural signs and symptoms of lithium intoxication are well documented. ${ }^{1}$ We report what we believe to be the first reported case of photophobia in this condition.

\section{Case report}

A 32-year-old woman with a 10-year history of manic-depressive psychosis was admitted to hospital after the rapid onset of a severe depressive episode. She also had polycystic ovary syndrome with amenorrhoea, obesity, hirsutism, acne, and infertility. She had been treated with lithium for four years and at the time of admission was taking $1600 \mathrm{mg}$ at night. To maintain a stable lithium concentration it was decided to change the dose to $800 \mathrm{mg}$ twice daily. After four days lithium concentration was $1.2 \mathrm{mmol} / 1(8.3 \mathrm{mg} / \mathrm{l})$ and dosage was altered to $600 \mathrm{mg}$ in the morning and $800 \mathrm{mg}$ at night. Three days later she complained of polyuria, polydipsia, ataxia, nausea, abdominal discomfort, hot and cold "flushes," mild headaches, and photophobia. The photophobia was such that she would not open her eyes under normal ward illumination and experienced severe pain when light from an ophthalmoscope was shone in her eyes. Fine lateral nygstagmus was also observed. Neurological examination showed no other abnormality and there were no signs of meningism. Temperature, pulse, blood pressure, and respiration remained normal.

Lithium intoxication was diagnosed, confirmed by a serum lithium concentration of $1.6 \mathrm{mmol} / \mathrm{l}(11 \mathrm{mg} / \mathrm{l})$ and her rapid recovery over 24 hours on stopping lithium treatment. The only other drug being taken concurrently was thyroxine $0.1 \mathrm{mg}$ daily for hypothyroidism (secondary to lithium treatment) and no other drug had been recently withdrawn. Lithium was restarted at a lower dose and the patient was discharged well, free from side effects.

\section{Comment}

A search of the published reports has revealed no similar case reports, though Lewy ${ }^{2}$ has suggested that patients with manic depression may be hypersensitive to light. Some reviews have linked pineal function to both light sensitivity and mood regulation but this does not seem to explain the above observation. We would be interested to hear reports of any similar cases or to receive comments. 